Klaus Matwald

\title{
Kempowskis Einfache Fibel - »so einfach ... nicht«
}

\section{Zum biographisch-pädagogischen Hintergrund}

Wer sich für die Einfache Fibel interessiert, wird schnell auf den »Schulmeister" Kempowski (vgl. Neumann 1980) stoßen. Richten wir den Blick zunächst jedoch auf eine literarische Figur, die als Kempowskis pädagogisches alter ego gelten darf: Im Jahr 1983 erschienen Herrn Böckelmanns schönste Tafelgeschichten, gesammelte Lesestücke des freundlich-verschrobenen Lehrers, der bereits 1979 in einem fiktiven Kinderbericht als Unser Herr Bökkelmann aufgetreten war. In einer dieser Tafelgeschichten heißt es: "Ganz ehrlich: Die Schule ist nicht angenehm. Aber wir haben lesen gelernt, und das ist gut, denn sonst nützt uns die Fernsehzeitung nichts" (KeMPOwsKI 1983: 100).

In diesen lapidaren Sätzen scheint manches von dem auf, was den Schriftsteller Kempowski ausmacht und auch auszeichnet: eine lakonische, geradlinige Sprache; ein kommentarfreier Realismus; eine trockene, doch nicht feindselige Ironie. ${ }^{1}$ Die Textstelle zeigt weiter, dass, wie gerne in Kempowskis Werk, so auch in einer heimeligen Tafelgeschichte "das Abgründige und Skurrile" (Plöschberger 2006: 36) jäh durch die Fassade der Harmlosigkeit brechen kann. Und schließlich lässt sich die Textstelle auch als Warnung davor lesen, Aussagen literarischer Figuren geradlinig auf ihren Autor zurückzuführen. ${ }^{2}$ Denn dass Schule "nicht angenehm « sei, hat Kempowski stets mit Überzeugung, Engagement und Erfolg zu widerlegen getrachtet.

Wie das Werden und Wirken des Lehrers Kempowski biographisch motiviert, didaktisch und pädagogisch fundiert sowie unterrichtspraktisch

1 Dass auch Kempowski dem Fernsehen zugetan war, zeigt sich beispielsweise an dem in Bloomsday '97 (1997) dokumentierten Selbstversuch oder in Tagebuchauszügen aus dem Jahr 2001 (vgl. Arnold 2006: 8, 10, 11, 12 ff., 29). Auch Kempowskis literarische Doppelgänger Alexander Sowtschik aus Hundstage (1988) und Lehrer Böckelmann (1979: 48 und 87) sehen gerne fern.

2 Figurenreden dem Autor zuzurechnen und anzukreiden ist ein unseliger Topos in der Kempowski-Rezeption. Vgl. kritisch hierzu etwa BRAND 2006: 87. 
gestaltet war, kann hier nur skizziert werden. ${ }^{3}$ Frühe Eindrücke humaner Lehrerfiguren und erste Bildungserfahrungen im Kreis der Bautzener Mithäftlinge (vgl. Hempel 2004: 38, 77; Henschel 2009: 128-130) waren prägend für Kempowski. Sein Lehramtsstudium in Göttingen stand klar im Zeichen reformpädagogischer Ideen, als deren Protagonisten zu Beginn des 20. Jahrhunderts Berthold Otto, Hermann Lietz, Georg Kerschensteiner und Adolf Reichwein hervorgetreten waren. Der Rektor der Pädagogischen Hochschule in Göttingen, Heinrich Heise, vertrat im Geist der Reformpädagogik eine "entscholastisierte Schule«, die von "Liberalität und Menschenfreundlichkeit« geprägt sein sollte (vgl. Henschel 2009: 133 f.). Gegen die Wilhelminische Prügel- und Drillschule, wie sie in der brachialen Nachhilfelehrerin »Tante Anna« aus Tadellöser \& Wolff(1971) sowie in vielen Äußerungen aus Schule. Immer so durchgemogelt (1974) durchscheint, setzte die Reformpädagogik die Idee einer humanen Erziehung als fürsorglicher Entwicklung der kindlichen Individualität in der tätigen Auseinandersetzung mit konkreten Dingen. Die lebens- und naturnahe "Sachbegegnung ... vom Kinde aus" (Henschel 2009: $151 \mathrm{f}$.) erfolgte bevorzugt im fächerübergreifenden Gesamtunterricht, in Freiarbeit, in gemeinschaftlichen Projekten und im Erkunden außerschulischer Lernorte.

In Neumanns Foto-Dokumentation ist zu sehen, wie sehr sich Kempowski der Schulmeister (1980) diese Ideen zu eigen gemacht hat. Sein Unterricht greift auf, was die Kinder beim morgendlichen Erzählen bewegt (vgl. ebd.: 26, 49), und er legt Wert auf anschaulichen Sachbezug und produktives Tun: Türme werden gebaut, Collagen geklebt, in so genannten »Mischheften" wird gezeichnet, gemalt und geschrieben (vgl. ebd.: 80). Die Lust am Nonsens zeigt sich in abstrusen Gutscheinen, die es zur Belohnung gibt, z. B. "Schielgutschein" oder "Dicke Backen-Gutschein" (vgl. ebd.: 98 f.). Der Lehrer lädt die Kinder zu sich nach Hause ein; am Feldrand werden Getreidearten erklärt; bei einem »Leserundgang« zum Kaufmann, zur Tankstelle, zum Drogisten werden die neu erworbenen Lesefertigkeiten erprobt (vgl. ebd.: 93).

Den Bildungsreformen um 1970 steht Kempowski skeptisch gegenüber. Im Interview 1979 klagt er über »Listen, Karteien, Konferenzen, Absicherungen« und das neue "Übermaß an Bürokratie» (zit. nach Henschel

3 Ein Portrait des Lehrers Kempowski kann sich auf ergiebige Quellen stützen: Michael Neumann zeigt ihn 1980 ausführlich in einem Foto- und Textband; Dirk Hempels Biographie (2004: 90-172) zeichnet sein Pädagogik-Studium in Göttingen, seine Tätigkeiten ab 1960 in den Dorfschulen Breddorf und Nartum, ab 1974 in der "Mittelpunktschule« Zeven sowie die aus seiner Lehrertätigkeit erwachsenden Veröffentlichungen nach: den "Befragungsband "Schule. Immer so durchgemogelt (1974), die Böckelmann-Bücher (1979, 1983), die Einfache Fibel (1980) oder die Lesetexte Haumiblau. 208 Pfenniggeschichten für Kinder (1986). Gerhard Henschel widmet dem »Pädagogen« Kempowski ein eigenes Kapitel (2009: 128-153). 
2009: 135); im Tagebuch Sirius (1990) mokiert er sich über »[r]iesige Schulen, in die die deutsche Jugend hineingetrieben wird [und die sie] nach dreizehn Jahren Stumpfsinn in völliger Verblödung « verlässt (zit. nach HENSCHEL 2009: 150). Als die "Mittelpunktschule« seine Dorfschule ersetzt, sucht er das "Idealbild der guten deutschen humanen Landschule in die Stadt zu übertragen" (zit. nach Henschel 2009: 133; vgl. auch NeumanN 1980: 9). Zu diesem Idealbild gehören der Lehrer Böckelmann (1979, 1983) und die zumindest in pädagogischer Hinsicht Heile Welt (1998) des jungen Dorfschulmeisters Jänicke. Kempowski äußert seine Überzeugungen auch direkt: Er favorisiert den nicht-autoritären Lehrertyp in der Rolle einer "Kindergartentante" (vgl. Neumann 1980: 7; Damiano 2005: 182) und sieht die Reformpädagogik als »unglaublich segensreiche Erfindung ... für die Menschheit« (zit. nach Henschel 2009: 133). Das Erziehungsziel könne nur sein: "Der im Kind angelegten Individualität zum Durchbruch zu verhelfen. Daraus folgt: möglichst wenig eingreifen, viel Zeit lassen und ständig ermuntern" (zit. nach Lennemann 2000: 120 f.). Pädagogisches Handeln verdichtet sich letztlich auf "[1]ieb sein und die Interessen der Kinder befriedigen" (zit. nach Henschel 2009: 144).

Im Erstleseunterricht folgt Kempowski dem reformpädagogischen Credo eines situationsbezogenen und individualisierten Lernens. Bewusst arbeitet er ohne Fibel, favorisiert stattdessen freies Arbeiten mit der Erstellung einer Eigenfibel (vgl. Neumann 1980: 20; Lehnemann 2000: 113). Daher wäre zu fragen, warum es Kempowskis Einfache Fibel überhaupt gibt. Zunächst ist jedoch zu klären, was eine Fibel ist und wie Kempowskis Fibel beschaffen ist.

\section{Kempowskis Einfache Fibel (1980)}

Eine Fibel ist ein "geschlossenes Elementarbuch als Medium für den Leseund Schreiblehrgang im muttersprachlichen Anfangsunterricht" (Hofen 2006: 149). Erste Fibeln finden sich bereits im 16. Jahrhundert (z. B. von Valentin Ickelsamer 1527) und ebenso alt ist die Auseinandersetzung um ihre didaktische und methodische Ausrichtung.

\subsection{Die Fibel als Lernmedium}

Je nachdem, welchen Konzeptionen des Lesenlernens sie folgen - unterschieden werden gemeinhin die Buchstabier-, die Lautier- und die Ganzheitsmethode (vgl. Menzel 2001) -, lassen sich Fibeln bis in die 1970er 
Jahre unterteilen in synthetische und analytische: "Soll man mit den Elementen der Schrift, den einzelnen Buchstaben, beginnen und lehren, wie sie zu synthetisieren sind, oder soll man mit Sinneinheiten, Sätzen oder Wörtern, anfangen und lehren, wie sie zu analysieren sind « (MARENBACH 2006: 123) - so lautete die didaktische Gretchenfrage.

In Fibeln wauf synthetischer Grundlage« wie z. B. der Berliner Fibel (1967, 2. Aufl. 1975) werden aus Buchstaben und isolierten Silben einfache Wörter und Sätze zusammengesetzt. Kempowskis Fibel folgt hingegen konsequent einem analytischen, ganzheitlichen Prinzip. ${ }^{4}$ Der Leselehrgang beginnt nicht mit Buchstaben und Silben, sondern mit den Namen Renate und Willi sowie mit Wörtern für auch in einem Klassenzimmer naheliegende Dinge: Schrank, Fenster, Heizung, Tür (vgl. 3-5). ${ }^{5}$ Erst nachdem ein kleiner Wortschatz erarbeitet worden ist - Wo ist; der / die / das; am / an / auf; Schrank / Fenster / Heizung / Tür / Tisch / Wasser / Lampe / Haus / Auto / Baum; und; Vater / Mutter-, werden beginnend mit dem R/r die Buchstaben eingeführt, stets im Zusammenhang mit Wörtern und Texten, in denen sie gehäuft auftreten. Hier zum Vergleich das $S / s$ in der (synthetischen) Berliner Fibel und in Kempowskis analytischer Fibel (s. Abb. 1).

Sinnfällig ist nicht nur der Unterschied zwischen einem synthetischen und Kempowskis ganzheitlich-analytischem Ansatz, sondern auch die Bedeutung des Bildanteils, den in der Einfachen Fibel der Kinderbuchautor, Kabarett-Texter, Zeichner und Maler Manfred Limmroth (1928-2004) beisteuerte. Limmroth hatte sich seit den 1960er Jahren mit Karikaturen einen Namen gemacht, die u. a. in der Welt, der Zeit und im Stern erschienen. Er war Gründungsmitglied des Magazins Twen, arbeitete als Bühnenbildner und Texter des Düsseldorfer Kabaretts Das Kom(m)ödchen und veröffentlichte über 50 Bücher, besonders erfolgreich das Kinderbuch Das verschwundene Ottchen (1997). Manfred Limmroth war auch als abstrakter Maler tätig. Im Jahr 2008 ehrte das Wilhelm-Busch-Museum Hannover ihn mit einer Werkschau. ${ }^{6}$

4 Die aus den USA importierten Ganzheitsmethoden wurden in Deutschland von Artur Kern in den 1930er Jahren maßgeblich verbreitet und "bestimmten auch noch nach 1945 bis in die 60er Jahre den Anfangsunterricht im Lesen- und Schreibenlernen « (Hofen 2006: 151). Ganzheits- bzw. Wortbildmethoden sind für das Englische plausibler als für das lauttreuere Deutsche; in der heutigen Didaktik des Schriftsprach- und Orthographieerwerbs setzt man neben dem Lautbezug der Schrift (phonologisches Prinzip) sehr stark auf die Erkenntnis morphematisch und grammatisch erklärbarer Regelmäßigkeiten, die der Rechtschreibung zugrunde liegen.

5 Reine Seitenangaben beziehen sich im Folgenden auf Kempowski 1980.

6 Informationen zu Manfred Limmroth in: Hamburger Abendblatt vom 5. 7. 2004 unter http:/www.abendblatt.de/kultur-live/article262571/Zum-Tod-von-Manfred-Limmroth.html (9. 5. 2009); sowie in: life PR vom 4.6. 2008 unter http:// 

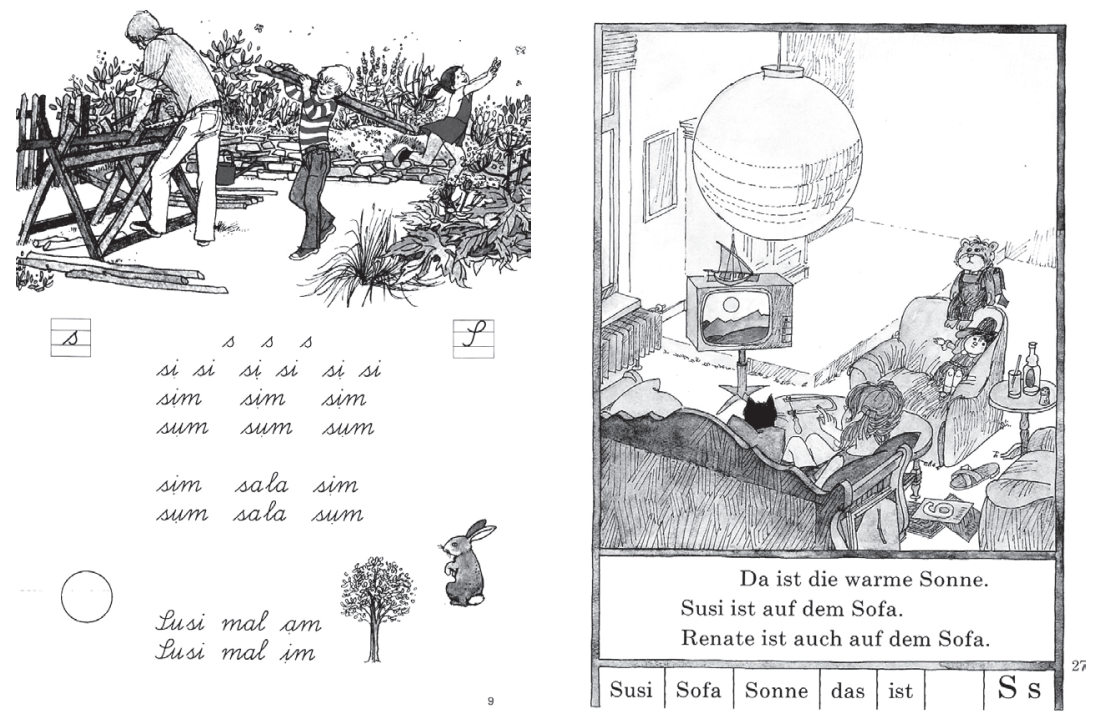

Abb. 1: Buchstabe $S / s$ in der Berliner Fibel (links) und in Kempowskis Fibel (rechts)

Fibelbilder intendieren neben ihren Funktionen für das Lesen und Schreiben $^{7}$ stets auch die "Vermittlung gesellschaftlicher Grundwerte und sozialer Verhaltensweisen " (Hofinger 2006: 150). Dass dies je nach Zeitgeist und weltanschaulichem Standort der Fibelmacher sehr unterschiedlich ausfallen kann (vgl. Grömminger 2001a, 2001b), zeigt sich auch hier: In der Berliner Fibel sehen wir Vater und Sohn beim tätigen Schaffen, während Susi etwas selbstvergessen Schmetterlingen nachhascht. (Dem Lehrer Böckelmann hätte diese Susi übrigens gefallen, war doch ein Pferdeschwanz »heutzutage schon selten geworden " [Kempowski 1979: 17].) In Kempowskis Fibel hingegen hat Renate offenbar erfolgreich die Fernsehzeitung gelesen und fläzt nun inmitten sachter Unordnung vor der Glotze. (Dass "die warme Sonne« medial aus dem Fernseher strahlt, soll schon hier als kleiner ironischer Bruch verbucht werden.)

www.lifepr.de/pressemeldungen/wilhelm-busch-gesellschaft-hannover-e-v/boxid47078.html (9. 5. 2009).

7 Dies wären die mnemotechnische Stützfunktion, die sinnerschließende Funktion, die motivationale Funktion, die sprachfördernde Funktion und die Schulung der optischen Differenzierungsfähigkeit (vgl. Grömminger/SCHIFfler 2001). 


\subsection{Die Einfache Fibel im Kontext der Didaktik um 1980}

Kempowskis Einfache Fibel erfüllt grundlegende Anforderungen der Textsorte: Sie verpflichtet sich konsequent einer methodischen Konzeption und wird von einem Lehrerband, einem Übungsbuch und 14 Ausschneidebögen zum Nachbasteln der Fibelwelt begleitet (Kempowski 1981a und b). Diese Ausschneidebögen sind sehr im Sinne reformpädagogisch erwünschter Konkretion, passen jedoch auch zu dem Kempowski, der seine verlorene Heimatstadt im Papiermodell nachbaute (vgl. Hempel 2004: 163). In der Progression der Fibel spiegelt sich der jahreszeitliche Ablauf des Schuljahres: Nach Wolken und Regen (26) gibt es Nebel (38 f.), sodann Advent / Weihnachten / Winter (40-49), Fasching (50 f.) und einen "Ferienausflug" an die See (75); »der letzte Schultag " (110) schließt das Buch ab. Der Textanteil schreitet von einfachen Wörtern und Sätzen über zusehends längere Reime, Geschichten und Märchen zu Beispielen aus der Alltagswelt (Plakate, Kleinanzeigen); realistische und phantastische Texte ergänzen einander. Somit verwandelt sich das Lernbuch »langsam in ein Lesebuch" (Lehrerband, zit. nach LeHNEMANN 2000: 118).

Wie es sich gehört, nimmt auch Kempowskis Fibel »kindliche Erfahrungswelt als Ausgangspunkt (GRÖMMINGER 2001b: 196). Wichtig hierfür sind die Identifikationsfiguren Renate und Willi, die in vielfältigen, für Kinder meist positiv besetzten Situationen gezeigt werden: beim Spielen und Toben, beim Fernsehen und Lesen, bei Ausflügen in den Zoo und an die See, mit den Haustieren Susi und Rolf. (Nur am Rande taucht hingegen die Schule auf; vgl. 45-47.) Lustige Bilder und Texte steigern die Attraktivität des Buches für die kindlichen Leser: Ein Pinguin fährt Schlittschuh, und ein Walross bricht durch das Eis (43); „Opa hat keinen Zahn. Der Vogel hat auch keinen Zahn" (34). Zeitgemäß sehen wir eine Kernfamilie mit einem durchaus präsenten Vater; Oma und Opa, auf die als phonologisches Minimalpaar keine Fibel verzichten kann, wohnen im eigenen Haus (vgl. 18).

Darüber spart die Fibel problemträchtige Wirklichkeiten keineswegs aus. Diese liegen nicht nur im engeren kindlichen Erfahrungsbereich Zahnweh und Kranksein (35, 99), eine kalte Heizung (71), ein Hausbrand in der Nachbarschaft (54) -, sondern umgreifen auch gesellschaftliche Problemlagen. Um 1980 sind dies, zeitgeschichtlich erwartbar, die Knappheit von Öl und Benzin (vgl. 70), Ärger mit »Autos« und »Verkehr» (64 f., 86 f.) und Fragen der Müllentsorgung (vgl. 73, 96).

Aussagen Kempowskis über die Einfache Fibel hat Widar Lehnemann (2000) zusammengetragen. ${ }^{8}$ Die Ganzheitsmethode sei die einfache, weil

8 Sie stammen aus dem Lehrerband, aus Tagebucheinträgen v. a. in Sirius (1990) und aus einem Vortrag über Lesenlernen - trotz aller Methoden. Ein Exkurs über Fibeln 
die Kinder an einfachen Wörtern für anschauliche Dinge spielerisch die Schrift erlernten (vgl. ebd.: 112 f.); die Fibel wolle kein »Zurichtungsinstrument « für die »Technik des Lesens", sondern ein »richtiges Buch« für »interessierte Leser" sein (vgl. ebd.: 114, 117); hierfür knüpfe sie an »Erfahrungen von Kindern« an (ebd.: 117) und spare "zeitkritische Texte" bewusst aus (vgl. ebd.: 120). Dass Kempowskis Fibel kritische Reaktionen erntete und auch keine Zulassung als Lernmittel erhielt (vgl. LeHnemann 2000: 119; Hempel 2004: 166), verwundert indes wenig. Nicht nur scheint die dargestellte Fibelwelt recht idyllisch und traditionell, auch war die Ganzheitsmethode um 1980 definitiv überholt, und die Fibel geriet als Lernmedium allmählich generell in die Kritik. ${ }^{9}$ Des Weiteren legt ein Blick in Neumanns Dokumentation nahe, dass Kempowski in der Fibel sehr stark den eigenen Unterricht nachbildete. Wir sehen dort Fotos, auf denen Kinder Kärtchen mit den Wörtern Heizung, Tisch oder Wasser an entsprechenden Gegenständen im Klassenzimmer anbringen oder auf denen Türme aufgerichtet bzw. an die Tafel gezeichnet werden (vgl. Neumann 1980: 20 f., 32-35). Heizung, Tisch und Wasser werden auch in der Fibel zu Beginn eingeführt (s. u. Abb. 2), ebenso gibt es dort für das T/t einen Turmbau (25). Von der Tafel seines Klassenzimmers in die Fibel übernommen hat Kempowski auch die Geschichte von der Dame mit den drei Dackeln (vgl. Neumann 1980: 53; Einfache Fibel: 68).

(1987). - Nach der Ganzwortmethode arbeiten auch Lehrer in den Romanen Schöne Aussicht (vgl. Lehnemann 2000: 146) und Heile Welt (vgl. Kempowski 1998: 365).

9 Um 1980 verfolgten Fibeln meist einen integrierten analytisch-synthetischen Ansatz. Dieser geht (analytisch) von der Wort- und Lautanalyse aus, ordnet dann (synthetisch) Laute und Buchstaben einander zu und baut das Wort durch Schreiben auf (vgl. Hartinger 2005: 65). Überdies trat an die Stelle eines separat vorauslaufenden Erstlesens zusehends die Idee eines Lesen und Schreiben integrierenden Schriftspracherwerbs. In diesem Kontext geriet das von einer Fibel instruierte kleinteilige Lernen im Gleichschritt generell in die Kritik, weil es neueren Auffassungen über heterogene Lernvoraussetzungen und Lernen als subjektivem Konstruktionsprozess widersprach. Diesen Vorstellungen suchten alternative Formen eines Schriftspracherwerbs ohne Fibellehrgang Rechnung zu tragen, etwa Jürgen Reichens Konzept eines selbstgesteuerten Lesens durch Schreiben (nach Anlauttabelle), Christa Röber-Siekmeyers aktives Erforschen orthographischer Regelmäßigkeiten oder Hans Brügelmanns "Spracherfahrungsansatz«, der den Schriftspracherwerb analog zum Sprechen in natürlichen Verwendungssituationen verortet (vgl. zusammenfassend BERGK 2001; zur Kritik an Reichen und am Spracherfahrungsansatz auch Buck 2001). Heute geht man davon aus, dass insbesondere Lernern aus schriftfernen Milieus ein enger geführter synthetischer Fibellehrgang besser entspricht und dass Fibeln generell zur Differenzierung und Individualisierung dienlich sein können (vgl. Bergk 2001: 397; Hartinger 2006: 65). 
Vielleicht ist daher die Vermutung nicht abwegig, dass es Kempowski weniger um ein marktfähiges Lernmittel und die Verbreitung lesepädagogischer Ideale zu tun war als darum, von seiner Arbeit etwas Bleibendes zu hinterlassen. Für diese Vermutung sprächen auch die Spuren von Kempowskis Gedankenwelt und Weltbild in der Einfachen Fibel.

\subsection{Spuren von Kempowskis Gedankenwelt und Weltbild in der Einfachen Fibel}

Zum einen führt uns Kempowskis Fibel eine traditionelle, bürgerliche $F a$ milie vor: Die Eltern und die Kinder im Reihenhaus sind flott und pfiffig, sie entsprechen im Kern aber der von Herrn Böckelmann beschriebenen "Altmodischen Familie«: "Da fährt der Vater morgens zur Arbeit, und die Mutter bleibt zu Hause (Kempowski 1983: 34). Der Fibelvater verlässt das Haus mit Jackett und Aktentasche, die Mutter organisiert Kindergeburtstage (89 f.) und malt Bilder (32). ${ }^{10}$ Die Kinder wirken gleichberechtigt, und doch zieht Renate einen Wagen mit Puppen und Plüschtieren, wo Willi lässig auf einem Roller sitzt (14 f.), und kocht Renate, wo Willi ein Segel hisst (59). Das Wohnzimmer enthält typische Akzidenzien einer bildungsorientierten Mittelschichtfamilie (s. Abb. 2).

In die bürgerliche Wohnstube gehören Weichholzschrank und Zimmerpflanzen. Die Bilder zeigen moderne und gegenständliche Motive - darunter das Brandenburger Tor. Auffällig sind Anzeichen diverser literaler Aktivitäten: Bücher auf dem Couchtisch, eine Zeitung, ein gut gefülltes Bücherregal, ein offener Sekretär.

Zweitens durchzieht Kempowskis Fibel eine unterschwellige Fortschrittskritik und sanfte Nostalgie. Nicht überraschend enthält das Wohnzimmer Modelle und (links über dem Sofa) das Gemälde eines Segelschiffs. Auch Opa und Oma spielen in ihrem heimeligen, von einem Holzofen beheizten Häuschen mit Spielzeugmodellen, in ihrem Speicher stehen alte Möbel und Spielsachen umher (18 f.). Zum Einkaufen (84 f.) sucht Willi einen pittoresken Krämerladen auf. »Dort wird ein Baum abgesägt« (ebd.), anderswo verfällt ein altes Bauernhaus zwischen Neubauten und Autobahn. Als nostalgisch kann auch der ausgeprägte Bezug auf die Textsorte Märchen gelten (vgl. 80, 90 f., 100, 102 f.).

10 In einem Tagebucheintrag 1983 mokiert sich Kempowski über die Kritik einer »Junglehrerin ... daß die Mutter kein Auto hat ... Und wieso soll die Mutter das Öl bestellen, wieso tut der Vater das nicht?» (zit. nach LeHnemann 2000: 119). 

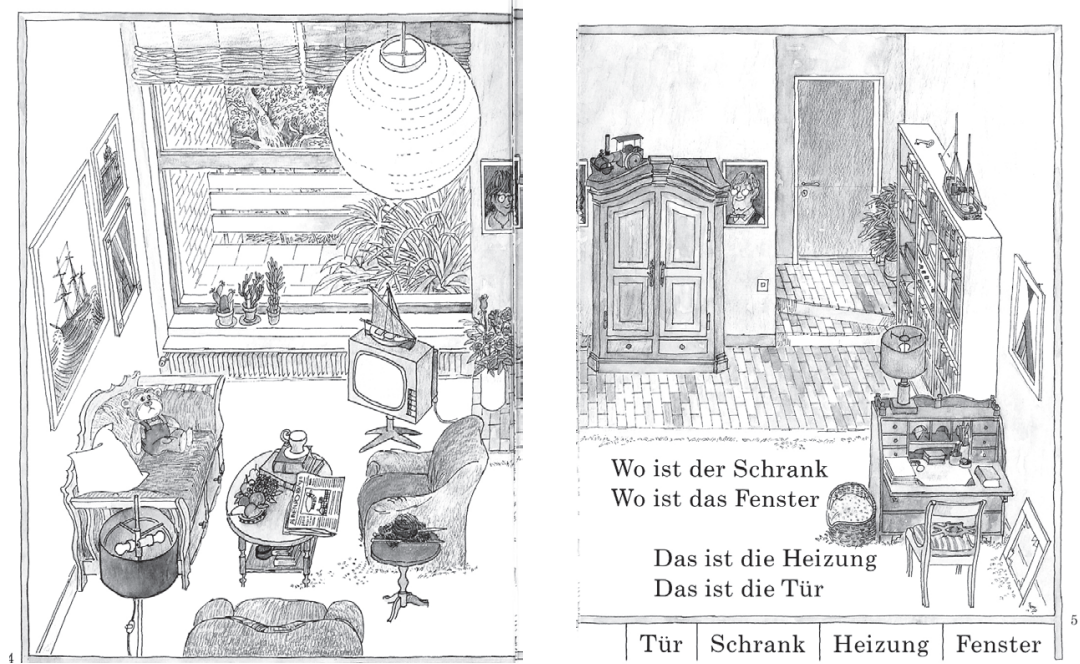

Abb. 2: Bürgerliches Wohnmilieu in Kempowskis Fibel (4 f., Ausschnitt)

Wie erwähnt, zeigt sich in der Fibelwelt der Ernst des Lebens mit Zahnarzt, Müllabfuhr und Feuerwehr. Vor allem aber tun sich, wie oft bei Kempowski, in der vordergründig heilen Welt immer wieder Risse auf. Auf dem Autobahnkreuz gibt es im fließenden Verkehr eine Reifenpanne und einen Unfall (65), aus dem Schlauch des Tankwagens tritt beiläufig Heizöl aus (71). Bereits in die erste Wohnzimmeransicht hat sich ein heruntergefallener Bilderrahmen geschlichen (vgl. Abb. 2 rechts unten). Etwas grotesk mutet auch die zugewucherte und vernachlässigt wirkende Vorderfront der Reihenhauszeile an (10 f.; vgl. auch 16). So ganz "einfach" und "in Ordnung" (vgl. Lehnemann 2000: 119) ist diese Fibelwelt nicht.

Jörg Drews schätzte an Kempowskis Tagebüchern den »wohldosiert beigegebenen erheiternden Blödsinn" (Drews 2006: 52). Auch hier gesellen sich zu den Rissen in den Bildern komische bis absurde Texte, meist in Reimform: »die Suppe | ist der Puppe | schnuppe« (67), oder: »Vom Dach | in den Bach | das macht wach« (77), oder folgender Abzählreim:

Auf einem Gummi-Gummi-Berg, da saß ein Gummi-Gummi-Zwerg, der aß ein Gummi-Gummi-Brot, da war er gummi-gummi-tot. (67)

Zum Glück geht Essen nicht immer tödlich aus: 
Ich bin Klein-Klaus,

ich wohne zu Haus,

ich kann nicht nähen,

ich kann nicht säen,

doch was statt dessen?

Wurst kann ich essen! (104)

Die abschließenden Doppelseiten simulieren den "Leserundgang«, den Kempowskis Schüler und Schülerinnen stets am Ende des Schuljahres unternahmen. Bereits die Schilder und Plakate von der Briefmarke bis zum Autobahnwegweiser wirken - auch in der disparaten Collage - komisch (106 f.). Vollends in den Nonsens drehen die fingierten Kleinanzeigen (108 f.), die u. a. eine 50 Meter lange Parkbank, die Stelle eines Opernsängers, »der Geige spielt und auch ein wenig frisieren kann", sowie einen verlorenen Zwiebelring und einen kombinierten »Eierlöffelbecher» annoncieren:

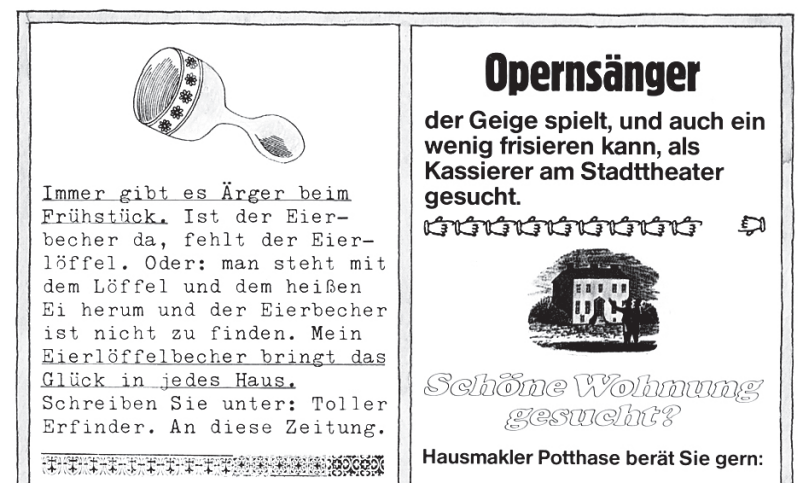

Abb. 3: Nonsens in Kempowskis Fibel (109, Ausschnitt)

Lakonische Ironie und ein Zug ins Groteske kennzeichnen auch die Romane Kempowskis. Die Fibelzeigt keinen »schrecklichen Kontrast zwischen Fassade und Wahrheit«, den Jörg Drews (2005: 16) vermerkt hat, wohl aber Risse in der "scheinbare[n] Behaglichkeit [und] stellenweise[n] Niedlichkeit« (ebd.). Ähnliches gilt für den wertungsfreien und doch differenzierenden Blick. Das von Carla Damiano notierte "Fehlen eines Kommentars" in Kempowskis Werk (Damiano 2005: 185) gilt auch in der Einfachen Fibel. An alte Zeiten wird erinnert, ohne sie zu verklären, und die moderne Gegenwart wird weder bejubelt noch denunziert. Wie Peter Brand für Heile Welt nachgewiesen hat (ebd.: 90), sind auch hier die Dinge, wie sie eben sind: nicht schwarz oder weiß, sondern mit vielen Zwischentönen. Zwei Beispiele mögen dies bezeugen. Ein Fibeltext warnt, dass man »Brot« nicht wegwerfen darf. Wenn es schimmelig ist, gibt man es »dem Nachbarn für seine Schweine. Wenn 
der Nachbar aber keine Schweine hat? Dann hat er vielleicht Hühner. Und wenn er keine Hühner hat? Na, dann weiß ich auch nicht« (95). Ein anderer Text handelt - in dialektischer Finesse - von »Nachbarn» (98):

Es gibt gute, und es gibt böse Nachbarn.

Böse Nachbarn stellen das Fernsehen zu laut an, sie laufen einfach durch unseren Garten und sagen nicht guten Tag.

Gute Nachbarn lächeln, wenn man sie sieht. Sie passen auf die Kinder auf und leihen einem auch mal einen Löffel Essig, wenn man gerade keinen Essig hat.

Wir selbst sind auch gute und böse Nachbarn, je nachdem. (98)

Viel Hintersinn liegt in diesen Fibeltexten - und viel von dem, was den Schriftsteller Kempowski ausmacht.

\section{Schluss: Die Einfache Fibel als Werk und im Werk des Schriftstellers Kempowski}

An einer wunderbaren Stelle des Romans Heile Welt lässt sich der Junglehrer Jänicke über den Referenten einer Fortbildung aus. Der Deutschdozent, so Jänicke, "war ein richtiger Germanist, sogar irgendwie Professor, wenn auch $\mathrm{PH}$, also irgendwie nur zum Professor ernannt, und sich nicht durch diverse Schikanen gequält" (Kempowski 1998: 433). Auch wenn man nicht "PH« ist, nagt am Didaktiker doch stets der Zweifel, ob man als »richtiger Germanist« durchgehen könne. Denn richtige Germanisten wie Jänickes Dozent beschäftigen sich mit gewichtigen Dingen wie einer "Chronopse der frühen Schiller-Werke, er wollte wissen, was Goethe, Wieland und Herder an ein und demselben Tag gemacht haben wie Schiller, zur selben Stunde möglichst sogar?« (Ebd.)

Die Beschäftigung mit einem Büchlein für ABC-Schützen kann da nur schwer mithalten. Nun hoffe ich aber gezeigt zu haben, dass die Einfache Fibel nicht nur als lesepädagogisches Dokument von Belang ist, sondern auch als Ausdruck der Gedankenwelt des Literaten Kempowski: die traditionelle Bürgerlichkeit, die Spannung zwischen Vergangenheit und Gegenwart, die Brüche in einer scheinbaren Idylle, der Zug ins Ironische und Groteskewas in Kempowskis literarischem Werk schwingt, kommt auch hier zum Klingen. Ich würde daher die Einfache Fibel nicht nur im Kontext der "Spiegelungen des Schulmeisters" sehen (vgl. LeHnemann 2000: 146), ${ }^{11}$ sondern

11 Lehnemann zeigt, dass die Tafelgeschichten (1983) eine Art Fortsetzungslesebuch zur Fibel sind, dass es Parallelen zwischen den "Pfenniggeschichten " in Haumiblau (1986) und der Fibel gibt und dass neben dem Lehrer Böckelmann auch weitere Lehrerfiguren wie Herr Jonas aus Schöne Aussicht (1981) oder Matthias Jänicke aus Heile Welt (1998) dem Schulmeister Kempowski nachempfunden sind. 
weitergehend als Werk im Werk des Schriftstellers. Kempowski selbst betonte die Personalunion: "Für mich ist es im Grunde dasselbe, ob ich jetzt Bücher schreibe, also Erwachsenen etwas erzähle, oder ob ich Kindern ein Märchen erzähle oder ihnen eine Sache klarmache, sagen wir die Wasserpumpe« (zit. nach BRAND 2006: 91). Hierzu passt seine Einschätzung, man müsse »das, was man lehrt, für die Schüler erfahrbar machen. Daran halte ich mich auch als Schriftsteller" (zit. nach Henschel 2009: 152). ${ }^{12}$

Dieser Konnex lässt sich über Entsprechungen im Inhalt und in den Motiven hinaus noch weiterdenken. Die Arbeit des Schriftstellers Kempowski ist vielfach als die eines bloßen Sammlers und Archivars abgetan worden (vgl. im Überblick ebd.: 22 f., 30, 36, 47, 51). Erst vor dem Hintergrund eines üppigen Gesamtwerkes aus Romanen, Befragungsbänden, Tagebüchern, Schulgeschichten sowie den monumentalen Echolot-Collagen wurde Kempowski erkennbar als ein "Beobachter, der mittels der Literatur eine historische Zeit abbildet, bestimmte Verhältnisse zeigt, Denken und Handeln des Bürgertums vorführt " (Hempel 2004: 131) - und der sich dabei immer wieder der eigenen Biographie versichert.

Ich schlage vor, die kleine Fibel im Gesamt dieses großen Projekts zu sehen. Da Kempowski ab 1981 bis zur Pensionierung an die Universität beurlaubt war, hat er seine Fibel selbst kaum noch einsetzen können. Da er dezidiert ohne Fibel arbeitete, ist sein pädagogischer Antrieb ohnehin etwas rätselhaft, zumal für dieses allzu eigene Erstlesebuch kaum Hoffnung auf Zulassung als Lehrwerk bestand. Was Kempowski mit der Einfachen Fibel in jedem Fall geschaffen hat, ist etwas Bleibendes vom stets nur flüchtigen Wirken eines Lehrers und das Dokument einer biographischen Wegstrekke. So gesehen wäre die Fibel ein unauffälliges, aber passendes Rädchen in dem Werk, das der Schriftsteller Kempowski ein Leben lang akribisch und beharrlich betrieben hat. Für dieses Werk aber und damit auch für die Einfache Fibel gilt, was darin vom Klavier zu lesen ist:

Ein Klavier ist sehr praktisch.

Aber so einfach,

wie man denkt,

spielt es sich nicht. (97)

12 Auch in der Forschung sind die Kontinuitäten zwischen dem Lehrer und dem Schriftsteller vermerkt worden. Dirk Hempel sieht bereits in den "Schul- und Kindergeschichten eine Anwendung des Prinzips der Fremderzähler« (Hempel 2005: 31), Carla Damiano (2005: 184) in Montage und Collage sowohl ein pädagogisches Werkzeug als auch ein literarisches Prinzip. 


\section{Literatur}

\section{Primärtexte}

Behrendt, Martin (1967) (Hg.): Berliner Fibel. Ein Lese- und Arbeitsbuch für den Anfangsunterricht auf synthetischer Grundlage. Druckschriftausgabe. Hannover: Schroedel (2. Aufl. 1975).

Kempowski, Walter (1971): Tadellöser \& Wolff. Ein bürgerlicher Roman. München: Hanser.

Kempowski, Walter (1974): Schule. Immer so durchgemogelt. München: Hanser.

Kempowski, Walter (1979): Unser Herr Böckelmann. Hamburg: Albrecht Knaus.

Kempowski, Walter (1980): Kempowskis Einfache Fibel. Braunschweig: Westermann.

Kempowski, Walter (1981a): [Einfache Fibel] Übungsteil. Braunschweig: Westermann.

Kempowski, Walter (1981b): [Einfache Fibel] Die 14 Ausschneidebögen. Braunschweig: Westermann.

Kempowski, Walter (1981c): Schöne Aussicht. Hamburg: Albrecht Knaus.

Kempowski, Walter (1983): Herrn Böckelmanns schönste Tafelgeschichten. Hamburg: Albrecht Knaus.

Kempowski, Walter (1986): Haumiblau. 208 Pfenniggeschichten für Kinder. München: Knaus.

Kempowski, Walter (1990): Sirius. Eine Art Tagebuch. München: Knaus.

Kempowski, Walter (1997): Bloomsday '97. München: Knaus.

Kempowski, Walter (1988): Hundstage. München: Knaus.

Kempowski, Walter (1998): Heile Welt. München: Knaus.

\section{Forschungsliteratur}

Arnold, Ludwig (2006) (Hg.): Walter Kempowski. Auszüge aus dem Tagebuch 2001. In: Text + Kritik 169, 3-31.

Bergk, Marion (2001): Alternative Formen des Schriftspracherwerbs - das Ende der Fibel? In: Grömminger (Hg.), 391-403.

Brand, Peter (2006): Latente Wahrnehmungsschwäche? Die Literaturkritik und Walter Kempowskis Roman »Heile Welt«. In: Text + Kritik 169, 82-93.

Buck, Siegfried (2001): Fibel und Schriftspracherwerb heute. In: Grömminger (Hg.), 367-389.

Damiano, Carla A. (2005): Walter Kempowski: Lehrer und Schriftsteller. Das Montage- / Collage-Prinzip als Baustein des Unterrichts und des Schreibens. In: Damiano/ Drews/Plöschberger (Hgg.), 171-187.

Damiano, Carla A./Drews, Jörg/Plöschberger, Doris (2005) (Hgg.): „Was das nun wieder soll?» Von Im Block bis Letzte Grüße. Zu Werk und Leben Walter Kempowskis. Göttingen: Wallstein.

Drews, Jörg (2005): Opening Statement. In: Damiano/Drews/Plöschberger (Hgg.), 9-20. 
Drews, Jörg (2006): »Die Dämonen reizen - und sich dann blitzschnell umdrehen, als sei nichts«. Über Walter Kempowski. In: Text + Kritik 169, 44-52.

Grömminger, Arnold (2001) (Hg.): Geschichte der Fibel (Beiträge zur Geschichte des Deutschunterrichts 50). Frankfurt/M.: Lang.

Grömminger, Arnold/Schiffler, Horst (2001): Die Funktion der Illustration in der Geschichte der Fibel. In: Grömminger (Hg.), 77-97.

Grömminger, Arnold (2001a): Das Bild der Schule in der Fibel. In: Grömminger (Hg.), 99-108.

Grömminger, Arnold (2001b): Die Fibeln nach dem 2. Weltkrieg bis zur Gegenwart. In: Grömminger (Hg.), 187-200.

Hartinger, Andreas (2005): Schriftspracherwerb - Erste Erfahrungen mit der Schriftsprache. In: Abraham, Ulf et al.: Praxis des Deutschunterrichts. Donauwörth: Auer, 63-72.

Hempel, Dirk (2004): Walter Kempowski. Eine bürgerliche Biographie. München: btb.

Hempel, Dirk (2005): Autor, Erzähler und Collage in Walter Kempowskis Gesamtwerk. In: Damiano/Drews/Plöschberger (Hgg.), 21-33.

Henschel, Gerhard (2009): Da mal nachhaken: Näheres über Walter Kempowski. München: dtv.

Hofen, Nikolaus (2006): Fibel. In: Kliewer, Heinz-Jürgen/Pohl, Inge (Hgg.): Lexikon Deutschdidaktik (Bd. 1). Baltmannsweiler: Schneider, 149-152.

Lehnemann, Widar (2000): Kempowskis Einfache Fibel. Text und Kontext. In: Ladenthin, Volker (Hg.): Die Sprache der Geschichte. Beiträge zum Werk Walter Kempowskis. Eitorf: gata, 107-149.

Marenbach, Dieter (2006): Erstlesen/Erstschreiben. In: Kliewer, Heinz-Jürgen/Pohl, Inge (Hgg.): Lexikon Deutschdidaktik (Bd. 1). Baltmannsweiler: Schneider, 122127.

Menzel, Wolfgang (2001): Geschichte der Methoden des Lesenlernens. In: GröMMINGER (Hg.), 55-64.

Neumann, Michael (1980): Kempowski, der Schulmeister. Braunschweig: Westermann.

Plöschberger, Doris (2006): Der dritte Turm. Die Tagebücher Walter Kempowskis. In: Text + Kritik 169, 32-43. 